УДК 621.313 .322

\title{
ОПТИМАЛЬНОЕ ПРОЕКТИРОВАНИЕ БЕЗРЕДУКТОРНОЙ МАШИНЫ С ПЕРЕКЛЮЧЕНИЕМ ПОТОКА ДЛЯ ВЕТРОГЕНЕРАТОРА
}

\author{
Прахт Владимир Алексеевич1, \\ va.prakht@urfu.ru
}

\author{
Дмитриевский Владимир Александрович1', \\ vladimir.dmitrievsky@urfu.ru
}

Казакбаев Вадим Маратович¹,
vadim.kazakbaev@urfu.ru

1 Уральский Федеральный Университет, Россия, 620002, г. Екатеринбург, ул. Мира, 19.

\begin{abstract}
Актуальность исследования обусловлена возрастающей потребностью применения автономных гибридных электростанций, использующих возобновляемые источники с целью уменьшения потребления органического топлива. Улучшение рабочих свойств и уменьшение стоимости безредукторных ветрогенераторов, применяемых в составе таких электростанций, способствует улучшению их технико-экономических характеристик.

Цель: поиск эфрфективного подхода для оптимизации генератора с переключением потока для безредуктороной ветроустановки, позволяющего сократить применение вычислительных ресурсов, в сравнение с существующими подходами; выработка рекомендаций к проектированию ветрогенератора с переключением потока с постоянными магнитами на статоре; улучшение характеристик конструкции генератора, спроектированной ранее.

объекты: математическая модель и конструкция генератора с переключением потока с магнитами на статоре, математическая модель безредуктороной ветротурбины.

Методы: безградиентный метод оптимизации, математическое моделирование, двухмерный метод конечных элементов, оценка и анализ скорости ветра в различных мировых регионах, статистические методы.

Результаты. Произведена многокритериальная оптимизация конструкции безредуктороного ветрогенератора с переключением потока. В результате были улучшены энергетические характеристики генератора, снижены пульсации момента, а также снижена номинальная мощность электронного преобразователя ветроустановки. Получены общие рекомендации, относительно геометрии конструкции, которые могут быть использованы при проектировании таких генераторов. Предложен метод расчета замещающих профилей для характеристики цикла работы ветротурбины, которой может быть использован для снижения вычислительных затрат в процессе оптимизации ветрогенераторов. Показано сокращение вычислительных затрат с помощью полученного метода на примере замены исходной девятиточечной характеристики цикла работы ветротурбины на замещающий двухточечный профиль.
\end{abstract}

\section{Ключевые слова:}

Автономные гибридные электростанции, безредукторные генераторы, ветрогенераторы,

ветроэнергетика, возобновляемые источники энергии, генераторы с постоянными магнитами,

оптимальное проектирование, электрическая машина с переключением потока, энергоэффективность.

\section{Введение}

Ввиду возрастающих цен на органическое топливо и трудностью его транспортировки для электроснабжения отдаленных, не связанных с единой энергосистемой, северных и восточных районов России все более актуальной становится использование гибридных генераторных установок. Такие установки наряду с ресурсами недр могут использовать возобновляемые источники: гидроресурсы рек, солнечную энергию и энергию ветра [1].

В связи с этим, в числе прочего, возрастает необходимость применения безредукторных ветряных турбин. Большое количество литературных источников посвящено оптимизации низкоскоростных ветрогенераторов для этого приложения [2-4].

В работе [5] рассматривается оптимизация безредукторного синхронного генератора с постоянными магнитами (ПМСГ) 500 кВт, 36 об/мин. Оптимизация проводится по критерию годовой выработки электроэнергии (ГВЭ). При этом учитываются 8 различных точек цикла работы генератора при разной скорости ветра (каждая точка цикла работы характеризуется различными величинами скорости вращения и механического момента на валу генератора). Отмечается, что применение такого критерия оптимизации вызывает очень большие затраты вычислительных ресурсов, поэтому оптимизационный расчет осуществляется с помощью распределенных параллельных вычислений.

В [6] рассматривается многокритериальная оптимизация маломощного (около 2 кВт, 250 об/мин) ПМСГ. В качестве критериев рассматриваются ГВЭ и масса магнитов. При этом учитываются 10 различных точек цикла работы генератора при разной скорости ветра. В работе используется генетический алгоритм. Целевая функция являлась отношением степенных функций критериев оптимизации (ГВЭ и масса постоянных магнитов).

В [7] рассматривается многокритерильная оптимизация ПМСГ большой мощности. В качестве критериев рассматриваются ГВЭ, масса и стоимость активных материалов. При этом учитываются 10 различ- 
ных точек цикла работы генератора при разной скорости ветра.

Все рассмотренные выше методы [5-7] позволяют оптимизировать конструкцию безредукторного генератора так, чтобы увеличить ГВЭ. Однако существенным недостатком данных методов является большая вычислительная трудоемкость, так как требуется рассчитывать характеристики генератора в 8 и более точках цикла работы в ходе каждой итерации.

В данной работе рассматривается методика построения замещающих профилей для характеристики цикла работы ветротурбины, содержащих небольшое число точек, например, 2 или 3. Методика применима как для упрощения дискретных характеристик цикла работы с большим числом точек, так и для дискретизации непрерывных характеристик. Этот подход открывает широкие возможности для оптимизации электрических машин, поскольку ресурсоёмкость расчёта целевой функции существенно уменьшается по сравнению с [5-7].

Достойной альтернативной традиционной конструкции ПМСГ с магнитами на роторе в рассматриваемом приложении может быть электрическая машина с переключением потока (ЭМПП) с магнитами на статоре, применение которой позволяет снизить расход редкоземельных постоянных магнитов [8-12] или полностью исключить их применение путем замены на магниты из менее дорогих материалов [11, 13].

В данной работе рассматривается критерий оптимальности для безредукторного генератора с переключением потока (ГПП), который направлен на увеличение ГВЭ (уменьшение потерь в генераторе), требуемой номинальной мощности AC/DC преобразователя, а также на уменьшение массы магнитов. Оптимизация ГПП с использованием предложенного критерия произведена с помощью метода Нелдера-Мида.

На основе предложенного метода в качестве примера спроектирован трехфазный безредукторый ГПП для ветротурбины; 9 рабочих точек, соответствующих максимальной мощности этой турбины для ряда скоростей вращения, взяты из [14]. Принято, что скорость ветра подчиняется статистическому распределению Релея [15]. На основе этой исходной девятиточечной характеристики построены двух- и трехточечные замещающие профили. Выполнена оптимизация с использованием двухточечного профиля. Вычисления, выполненные для исходной и оптимизированной конструкции по двух- и трехточечным замещающим профилям, сравниваются с вычислениями, выполненными по исходной девятиточечной характеристике/профилю. Показано, что результаты вычислений в этих трех случаях очень близки. Описываются основные геометрические соотношения оптимальной конструкции ГПП.

\section{Краткое описание выбранной конструкции ГПП и ее математической модели}

Рис. 1 показывает эскиз рассматриваемой конструкции ГПП с 24 зубцами статора и 22 зубцами ротора. Магниты вставляются в пазы, выполненные в каждом зубце статора. Магниты, расположенные в соседних пазах, имеют противоположное направление намагниченности. Целостность конструкции статора обеспечивается тонкими ребрами на внутренней и внешней поверхности. На каждом втором зубце статора размещается сосредоточенная катушка. Для снижения потерь от вихревых токов каждый магнит разделен на 3 изолированные части.

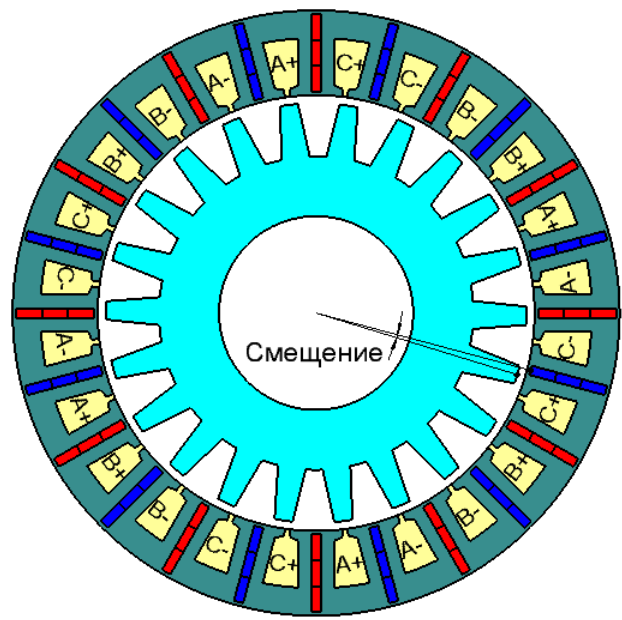

Pис. 1. Эскиз конструкиии 22/24 генератора с переключением потока

Fig. 1. Sketch of design of 22/44 flux switching generator

Математическая модель ГПП основана на решении последовательности двухмерных магнитостатических задач для диапазона различных позиций рото$\mathrm{pa}$, определяемого симметрией. Потокосцепления и напряжения фазных обмоток, потери в обмотках и магнитопроводе вычисляются после решения этих краевых задач методом конечных элементов (МКЭ). Принято, что фазный ток имеет синусоидальную форму [16-18]. Механическая мощность на валу генератора является исходным параметром для расчета. Амплитуда тока при этом рассматривается как величина, которую нужно подобрать для получения заданной механической мощности.

Электрическим периодом ГПП является поворот ротора на одно зубцовое деление, т. е. на $360 / 22=16,36^{\circ}$. Это значит, что частота питающего напряжения в 22 раза больше частоты вращения ротора $\left(f_{e}=Z_{r} \cdot n / 60\right.$ где $f_{e}$ - это электрическая частота статора, Гц; $n$ - механическая частота вращения ротоpa, об/мин; $Z_{r}$ - число зубцов ротора).

Пусть зубец ротора ГПП находится над серединой зубца статора, обмотанного катушкой фазы $A$. Поскольку число зубцов ротора близко к числу зубцов статора, в этот момент примерно над серединой ближайшего зубца статора, обмотанного фазой $C$, также находится зубец ротора, расположенный через 2 зубцовых деления ротора.

Более точный расчёт даёт величину смещения $2 \cdot 360 / 22-2 \cdot 360 / 24=360 /(22 \cdot 6)^{\circ}$ (рис. 1). Таким образом, ГПП обладает симметрией относительно поворота ротора на одну шестую электрического периода и перестановки фаз $I_{A} \rightarrow-I_{C}, I_{B} \rightarrow-I_{A}, I_{C} \rightarrow-I_{B}$. Поэтому достаточно рассмотреть положения ротора в интерва- 
ле 1/6 электрического периода. В данной работе этот интервал делится на 15 равных частей. Общее число рассматриваемых краевых задач при расчете характеристик машины равно 16, а именно краевые задачи для начала и конца интервала и ещё 14 промежуточных краевых задач.

Кроме того, симметрия конструкции машины, как целого, позволяет сократить расчётную область в 2 раза (рис. 2). В данном исследовании для всех краевых задач выбиралась одна и та же расчётная область. Расчётная область разделена на две подобласти линией, проходящей по середине воздушного зазора. Угол поворота ротора учитывается задаваемым вдоль этой линии сшивающим граничным условием, зависящим от времени. Границы $P$ I и $P$ II сшиваются периодическим условием.

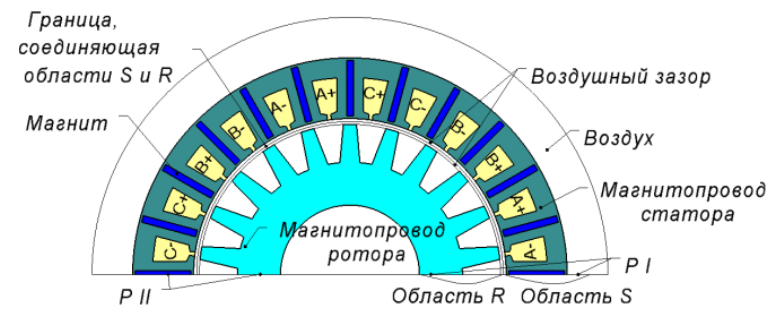

Puc. 2. Область расчета

Fig. 2. Calculation area

Чтобы учесть магнитное поле за внешней границей статора ГПП, добавлена воздушная область, толщина которой равна зубцовому делению статора. В целом представленная математическая модель ГПП аналогична математическим моделям других электрических машин, описанных в [19, 20].

\section{Расчет замещающих профилей нагрузки генератора}

Под профилем нагрузки генератора для ветряной турбины будем понимать совокупность зависимости момента $T$ от скорости вращения вала $n$, соответствующей максимальной мощности, и статистического распределения во времени точек характеристики рабочего цикла турбины. Профиль нагрузки может быть непрерывным. Тогда задается зависимость $T(n)$ и плотность распределения скорости $n$. Также профиль нагрузки может быть дискретным или заданным дискретно. Тогда задаются пары значения $T$ и $n$ и доля времени (вероятность), соответствующая этим значением $T$ и $n$.

Задание профиля нагрузки позволяет вычислять средние значения случайных величин. В частности, в случае дискретного профиля среднее значение 〈A> для любой случайной величины $A$ :

$$
\langle A\rangle=\sum p_{i} \cdot A_{i},
$$

где $A_{\mathrm{i}}-$ значение этой величины в $i$-й точке цикла работы; $p_{i}-$ вероятность $i$-й точки цикла работы.

Через средние механической мощности $P_{\text {mech }}=2 \pi \cdot n \cdot T / 60$ и мощности потерь $P_{\text {loss }}$ выражается средний КПД генератора:

$$
\eta_{\text {average }}=1-\frac{\left\langle P_{\text {loss }}\right\rangle}{\left\langle P_{\text {mech }}\right\rangle} .
$$

При большом числе точек в профиле вычисление средних по формуле (1) и, в частности, среднего КПД по формулам (1), (2) требует больших вычислительных ресурсов. Поэтому рассмотрим алгоритм построения замещающих профилей, содержащих меньшее число точек.

Будем считать, что скорость $n\left(P_{\text {mech }}\right)$ вращения и момент $T\left(P_{\text {mech }}\right)$ данного профиля являются функциями от механической мощности $P_{\text {mech }}$, определёнными на (непрерывном) интервале допустимых значений мощностей. Если эти зависимости задаются таблично, то значения скорости и момента для произвольной $P_{\text {mech }}$ устанавливаются путём интерполяции. В результате точка с произвольной $P_{\text {mech, }}$ а не только с её табличным значением, оказывается однозначно заданной. Поэтому произвольная случайная величина $A$ может также рассматриваться как функция от $P_{\text {mech }}$.

Пусть функция $A\left(P_{\text {mech }}\right)$ на интервале $P_{\text {mech }}$ аппроксимирована полиномом:

$$
A=a_{0}+a_{1} \cdot P_{\text {mech }}+a_{2} \cdot P_{\text {mech }}^{2}+\ldots .
$$

Операция $<>$ является линейной в том смысле, что

$$
\langle\alpha \cdot x+\beta \cdot y\rangle=\alpha\langle x\rangle+\beta\langle y\rangle,
$$

где $\alpha$ и $\beta$ - числа, а $x$ и $y$ - случайные величины. Из (3) и (4) следует:

$$
\langle A\rangle=a_{0}+a_{1} \cdot\left\langle P_{\text {mech }}\right\rangle+a_{2} \cdot\left\langle P_{\text {mech }}^{2}\right\rangle+\ldots .
$$

Таким образом, если полиномом $n$-й степени достаточен для аппроксимации $A$, то вычисление среднего $<A>$ по исходному профилю можно заменить вычислением того же среднего $<A>$ по замещающему профилю (5), в котором $\left\langle P_{\text {mech }}\right\rangle,\left\langle P_{\text {mech }}{ }^{2}\right\rangle \ldots\left\langle P_{\text {mech }}{ }^{n}\right\rangle$ совпадают с этими средними по исходному профилю.

Одной из точек цикла работы, которая обязательно должна быть рассмотрена, является точка с максимальной мощностью, поскольку необходимо рассчитать требуемую мощность AC/DC преобразователя и проверить отсутствие размагничивания магнитов.

Проблема размагничивания актуальна, в первую очередь, для машин, в которых используются низкокоэрцитивные постоянные магниты. Однако в работе [21] показано, что недопустимое размагничивание может иметь место и при использовании высококоэрцитивных «неодим-железо-бор» магнитов, если применяется синхронная машина. Поэтому при проектировании ГПП также необходима такая проверка.

Задание каждой точки с частичной нагрузкой

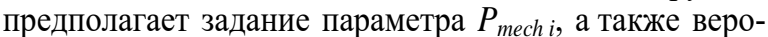
ятности $p_{i}$. Поэтому для вычисления среднего случайной величины $A$, для аппроксимации которой достаточно полинома $2 k$-й степени, достаточно использовать замещающий профиль с $k$ точками частичной нагрузки. В частности, значения $\left\langle P_{\text {mech }}>\right.$, полученные как по исходной характеристике цикла работы, так и по любому замещающему профилю, содержащему хотя одну точку частичной нагрузки, совпадают.

Уравнения для нахождения $2 k$ неизвестных $p_{1} \ldots p_{k}$, $\left(P_{\text {mech }, 1}\right)^{i} \ldots\left(P_{\text {mech }, k}\right)^{i}$ имеют вид:

$$
\begin{gathered}
\sum_{m=1}^{k}\left[p_{m} \cdot P_{\text {mech }, m}^{i}+P_{\text {rated }}^{i}\left(1-\sum_{m=1}^{k} p_{m}\right)\right]= \\
=\left\langle P_{\text {mech }}^{i}\right\rangle ; i=1 \ldots 2 k,
\end{gathered}
$$


где $P_{\text {rated }}$ - механическая мощность в точке с максимальной нагрузкой.

Когда квадратичной аппроксимации достаточно, т. е. в случае замещающего профиля с одной номинальной точкой и одной точкой частичной нагрузки (двухточечного профиля), можно получить простые аналитические решения (6):

$$
\begin{gathered}
P_{\text {mech }, 1}=\frac{P_{\text {rated }}^{2}-\left\langle P_{\text {mech }}^{2}\right\rangle}{P_{\text {rated }}-\left\langle P_{\text {mech }}\right\rangle}-P_{\text {rated }}, \\
p_{1}=\frac{P_{\text {rated }}-\left\langle P_{\text {mech }}\right\rangle}{P_{\text {rated }}-P_{\text {mech }, 1}} .
\end{gathered}
$$

В случае аппроксимации четверной степени (трехточечного профиля), решение нелинейных уравнений (6) может быть найдено любым методом из известных, например, методом Ньютона.

После нахождения механических мощностей в точках частичной нагрузки замещающего профиля скорости и моменты в этих точках определяются при помощи функциональных зависимостей $n\left(P_{\text {mech }}\right)$ и $T\left(P_{\text {mech }}\right)$.

\section{Пример расчета замещающих профилей содержащих 2 и 3 рабочих точки}

В данной работе в качестве примера рассматривается турбина, описанная в [14]. В работе [14] приводится механическая мощность, а также скорость вращения турбины в 9 точках, соответствующих скоростям ветра от 4 до $12 \mathrm{~m} / \mathrm{c}$. Пусть средняя скорость ветра $7 \mathrm{~m} / \mathrm{c}$. Для описания годового распределения скорости ветра широко используются статистические распределения Релея и Вейбулла [15], [22]. В данной работе было сделано допущение, что вероятность появления определенной скорости ветра соответствует распределению Релея, и что генератор работает при скоростях ветра в диапазоне от 4 до $12 \mathrm{~m} / \mathrm{c}$.

Чтобы совместить непрерывное распределение Релея с дискретными данными рабочего цикла (табл. 1), оно также было дискретизировано, т. е. считалось, что скорость ветра принимает целые значения м/с, вероятность той или иной скорости ветра равна плотности вероятности распределения Релея. Столбец $p_{i}$ табл. 1 указывает на условную вероятность, когда скорость ветра находится в допустимых пределах.

Найдем двухточечный и трехточечный замещающие профили. Требуемые средние величины, найденные по исходному профилю, приведены в табл. 2. Механические мощности в точках частичной нагрузки и вероятности находятся по формулам (6)-(8). На рис. 3 с помощью символа «»» показаны моменты и механические мощности в 9 точках исходного профиля. Также показан кубический полином, аппроксимирующий эту зависимость. С помощью аппроксимации этой зависимости находятся моменты, а затем скорости, соответствующие механическим мощностям двухточечного и трехточечного замещающих профилей (табл. 3, 4).

Двухточечный профиль приводится в табл. 3, а трехточечный - в табл. 4.
Таблица 1. Исходная девятиточечная характеристика иикла работы ветротурбины

Table 1. $\quad$ Operating points of the wind turbine (initial 9point profile)

\begin{tabular}{|c|c|c|c|c|c|}
\hline $\begin{array}{c}\text { Точка рабочего } \\
\text { цикла, } i \\
\text { Operating mode, } i\end{array}$ & $\begin{array}{c}V_{i}, \mathrm{M} / \mathrm{c} \\
(\mathrm{m} / \mathrm{s})\end{array}$ & $\begin{array}{c}n_{i}, \text { об/мин } \\
(\mathrm{rpm})\end{array}$ & $\begin{array}{c}P_{\text {mech, }, i}, \\
\mathrm{BT}(\mathrm{W})\end{array}$ & $\begin{array}{c}T_{i}, \mathrm{H} \cdot \mathrm{M} \\
(\mathrm{N} \cdot \mathrm{m})\end{array}$ & $p_{i}$ \\
\hline 1 & 4 & 111 & 82 & 7,02 & 0,134 \\
\hline 2 & 5 & 140 & 142 & 9,69 & 0,144 \\
\hline 3 & 6 & 163 & 237 & 13,87 & 0,146 \\
\hline 4 & 7 & 196 & 362 & 17,59 & 0,138 \\
\hline 5 & 8 & 221 & 542 & 23,41 & 0,124 \\
\hline 6 & 9 & 247 & 761 & 29,41 & 0,107 \\
\hline 7 & 10 & 276 & 1038 & 35,88 & 0,087 \\
\hline 8 & 11 & 308 & 1383 & 42,85 & 0,069 \\
\hline 9 & 12 & 332 & 1784 & 51,38 & 0,051 \\
\hline
\end{tabular}

Таблица 2. Средние значения механической мощности Table 2. Average values oh the mechanical power

\begin{tabular}{|c|c|c|c|}
\hline $\begin{array}{c}\left\langle P_{\text {mech }}\right\rangle, \mathrm{BT} \\
(\mathrm{W})\end{array}$ & $\begin{array}{c}\left\langle P_{\text {mech }}{ }^{2}\right\rangle, \\
\mathrm{BT}^{2}\left(\mathrm{~W}^{2}\right)\end{array}$ & $\begin{array}{c}\left\langle P_{\text {mech }}{ }^{3}\right\rangle, \\
\mathrm{BT}^{3}\left(\mathrm{~W}^{3}\right)\end{array}$ & $\begin{array}{c}\left\langle P_{\text {mech }}{ }^{4}{ }^{3}, \mathrm{BT}^{4}\right. \\
\left(\mathrm{W}^{4}\right)\end{array}$ \\
\hline 541,9 & $5,177 \mathrm{E}+05$ & $6,472 \mathrm{E}+08$ & $9,228 \mathrm{E}+11$ \\
\hline
\end{tabular}

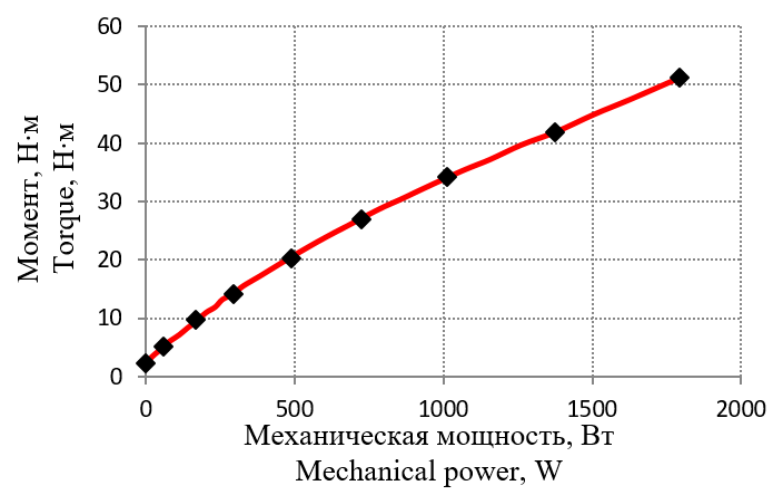

Pис. 3. Момент ветряной турбинь

Fig. 3. Wind turbine torque

Таблица 3. Двухточечных замещчающий профиль

Table 3. 2-point substituting profile

\begin{tabular}{|c|c|c|c|c|}
\hline $\begin{array}{c}\text { Точка рабочего } \\
\text { цикла, } i \\
\text { Operating mode, } i\end{array}$ & $\begin{array}{c}n_{i}, \text { об/мин } \\
(\mathrm{rpm})\end{array}$ & $\begin{array}{c}P_{\text {mech, }, \text {, }} \text { Вт } \\
(\mathrm{W})\end{array}$ & $\begin{array}{c}T_{i}, \mathrm{H} \cdot \mathrm{M} \\
(\mathrm{N} \cdot \mathrm{m})\end{array}$ & $p_{i}$ \\
\hline 1 & 193,6 & 361,6 & 17,84 & 0,873 \\
\hline 2 & 331,6 & 1784,3 & 51,38 & 0,127 \\
\hline
\end{tabular}

Таблица 4. Трехточечный замещающий профиль

Table 4. 3-point substituting profile

\begin{tabular}{|c|c|c|c|c|}
\hline $\begin{array}{c}\text { Точка рабочего } \\
\text { цикла, } i \\
\text { Operating mode, } i\end{array}$ & $\begin{array}{c}n_{i}, \text { об/мин } \\
(\mathrm{rpm})\end{array}$ & $\begin{array}{c}P_{\text {mech, }, \text {, } \mathrm{BT}} \\
(\mathrm{W})\end{array}$ & $\begin{array}{c}T_{i}, \mathrm{H} \cdot \mathrm{M} \\
(\mathrm{N} \cdot \mathrm{m})\end{array}$ & $p_{i}$ \\
\hline 1 & 161 & 206,3 & 12,24 & 0,623 \\
\hline 2 & 268,1 & 952,3 & 33,92 & 0,311 \\
\hline 3 & 331,6 & 1784,3 & 51,38 & 0,066 \\
\hline
\end{tabular}

\section{Процедура оптимизации генератора}

На рис. 4 изображены основные геометрические размеры ГПП. В табл. 5 приведены параметры ГПП, зафиксированные в ходе оптимизации. Здесь и далее угловые размеры статора приводятся в единицах зубцового деления статора $t_{s}=360 / 24=15^{\circ}$, угловые размеры ротора приводятся в единицах зубцового деления ротора $t_{r}=360 / 22=16,36^{\circ}$. Параметры ГПП, кото- 
рые варьировались в ходе оптимизации, и их начальные значения приведены в табл. 6.

При векторном управлении задание по вектору тока статора формируется в системе координат, вращающейся синхронно с ротором [23].

Под «углом тока» понимается угол между магнитной осью фазы $A$ и согласованным положением ротора: если один из зубцов ротора находится точно над серединой зубца статора, обмотанного фазой $A$, и ток фазы $A$ принимает максимальное значение, то при небольших токах достигается максимально возможное при данном модуле тока значение момента. Такое положение вектора тока относительно ротора будем характеризовать нулевым углом. Однако при увеличении тока с нулевым углом увеличивается реактивная мощность, что приводит к необходимости выбора более мощного AC/DC преобразователя. Увеличивается насыщение, что приводит к снижению КПД и момента, по сравнению с величинами КПД и момента, которые могли бы быть достигнуты при неизменном уровне насыщения. Изменение угла тока относительно нулевого положения в системе координат, связанной с ротором, позволяет смягчить эти явления. Поэтому угол тока задавался пропорциональным механической мощности генератора, и дополнительным параметром оптимизации являлся угол тока при максимальной нагрузке генератора.

Таким образом, под «ослаблением поля» понимается изменение угла тока относительно нулевого положения в системе координат, связанной с ротором. Выполняется такое «ослабление поля» для уменьшения приращения величины тока статора при возрастании момента генератора.

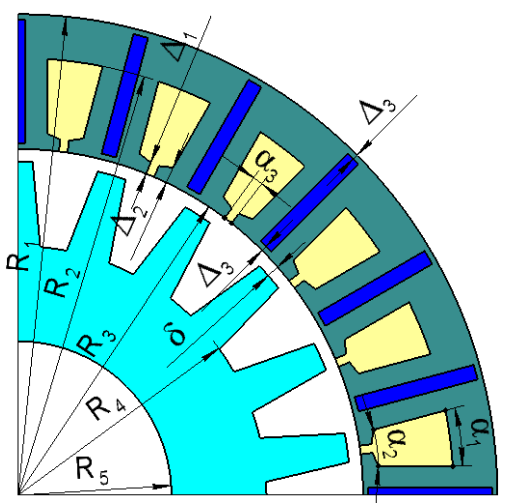

Pис. 4. Параметры оптимизаичии

Fig. 4. Optimization parameters

Многокритериальная оптимизация лучше всего подходит для проектирования электрических машин для реального приложения [24]. Некоторые методы оптимизации предназначены для расчета кривой Парето, которая является множеством решений, для которых ни один из целевых параметров оптимизации не может быть улучшен без ухудшения величин других целевых параметров. В этом случае инженер выбирает одно из решений, принадлежащих кривой Парето, исходя из требований и ограничений решаемой задачи.
Таблица 5. Постоянные параметры ГПП

Table 5. Parameters of the flux switching generator, constant during the optimization

\begin{tabular}{|c|c|}
\hline $\begin{array}{r}\text { Параметр } \\
\text { Parameter } \\
\end{array}$ & $\begin{array}{c}\text { Значение } \\
\text { Value }\end{array}$ \\
\hline $\begin{array}{l}\text { Внешний радиус статора } R_{1}, \text { мм } \\
\text { Outer radius of the stator } R_{1}, \mathrm{~mm}\end{array}$ & 80 \\
\hline $\begin{array}{l}\text { Воздушный зазор } \delta, \text { мкм } \\
\text { Air gap } \delta, \mu m\end{array}$ & 350 \\
\hline $\begin{array}{l}\text { Глубина паза ротора }\left(R_{3}-\delta-R_{4}\right), \text { мм } \\
\text { Depth of the rotor slot }\left(R_{3}-\delta-R_{4}\right), \mathrm{mm}\end{array}$ & 9 \\
\hline $\begin{array}{l}\text { Толщина ярма ротора }\left(R_{4}-R_{5}\right), \text { мм } \\
\text { Width of the rotor yoke }\left(R_{4}-R_{5}\right), \mathrm{mm}\end{array}$ & 5 \\
\hline $\begin{array}{l}\text { Толщина ребер статора } \Delta_{3} \text {, мкм } \\
\text { Width of the stator ribs } \Delta_{3}, \mu m\end{array}$ & 500 \\
\hline $\begin{array}{l}\text { Остаточная индукция магнитов, } T \\
\text { Remanent flux density of the permanent magnets, } T\end{array}$ & 1,2 \\
\hline $\begin{array}{l}\text { Угловой размер шлица паза статора } \alpha_{3}, t_{s} \\
\text { Angular size of the stator slot opening } \alpha_{3}, t_{s}\end{array}$ & 0,162 \\
\hline $\begin{array}{l}\text { Длина магнитопроводов ротора и статора } L, \mathrm{Mм} \\
\text { Length of magnetic cores of the rotor and the stator, } \mathrm{mm}\end{array}$ & 100 \\
\hline
\end{tabular}

Таблица 6. Параметры ГПП, изменяемые в ходе оптимизации

Table 6. Parameters of the flux switching generator, changed during optimization

\begin{tabular}{|c|c|}
\hline $\begin{array}{cc}\text { Параметр } \\
\text { Parameter } \\
\end{array}$ & $\begin{array}{c}\text { Значение } \\
\text { Value }\end{array}$ \\
\hline $\begin{array}{l}\text { Радиус дна паза статора } R_{2}, \text { мм } \\
\text { Radius of the stator slot bottom } R_{2}, \mathrm{~mm}\end{array}$ & 74 \\
\hline $\begin{array}{l}\text { Внутренний радиус статора } R_{3}, \text { мм } \\
\text { Inner radius of the stator } R_{3}, \mathrm{~mm}\end{array}$ & 61,7 \\
\hline $\begin{array}{l}\text { Угловой размер паза статора } \alpha_{1}, t_{s} \\
\text { Angular size of the stator slot } \alpha_{1}, t_{s}\end{array}$ & 0,45 \\
\hline $\begin{array}{l}\text { Угловой размер паза статора } \alpha_{2}, t_{s} \\
\text { Angular size of the rotor slot } \alpha_{2}, t_{s}\end{array}$ & 0,5 \\
\hline $\begin{array}{l}\text { Толщина магнитов, мм } \\
\text { Width of the permanent magnets, mm }\end{array}$ & 2 \\
\hline $\begin{array}{l}\text { Угловой размер поверхности зубца ротора, обращен- } \\
\text { ной к зазору, } t_{r} \\
\text { Angular size of the rotor tooth surface facing the air gap, } t_{r}\end{array}$ & 0,265 \\
\hline $\begin{array}{l}\text { Угол тока при максимальной нагрузке генератора, } \\
\text { электрических радиан } \\
\text { Current angle at the maximum generator power, electrical } \\
\text { radians }\end{array}$ & 0,1 \\
\hline
\end{tabular}

Другой подход к многокритериальной оптимизации может быть реализован на базе однокритериальных методов. В этом случае при составлении оптимизационной функции должны быть применены весовые коэффициенты, определяющие важность отдельных целевых параметров. При этом возможно использование как поощряющих, так и штрафующих коэффициентов [25].

Таким образом, при любом подходе к оптимизации важность отдельных целевых параметров должна быть определена либо в начале, либо в конце оптимизационной процедуры.

В данной работе на выбор оптимизационного алгоритма повлияли гладкость целевой функции и наличие вычислительной ошибки, возникающей из-за конечного числа рассматриваемых граничных задач при повороте ротора, а также из-за конечного размера элементов сетки. Эти факторы создают псевдослучайную ошибку, которую трудно предсказать. Величины целевой функции, вычисленные с помощью МКЭ, оказываются зашумлены, и градиентные мето- 
ды, применяемые обычно для гладких функций с малым числом экстремумов, не могут быть применены $[19,20]$.

Для преодоления этой трудности в данной работе используется метод Нелдера-Мида. Это безградиентный метод безусловной оптимизации функции нескольких переменных, который может быть успешно применен в случае негладких и/или зашумлённых функций. Метод широко используется для нелинейной оптимизации и хорошо подходит для задач, в которых производные функций неизвестны. В большинстве случаев метод Нелдера-Мида требует 1-2 вычислений целевой функции за 1 итерацию, что позволяет сократить число вычислений целевой функции при нахождении экстремума $[19,20]$.

Используется следующая целевая функция оптимизации:

$$
F=K_{1} \cdot K_{2}^{2} \cdot K_{3}^{0,5},
$$

где $K_{1}, K_{2}$ and $K_{3}$ - целевые параметры (критерии) оптимизации: $K_{1}=\left\langle P_{\text {loss }}>\right.$ применяется для увеличения КПД, ГВЭ генератора $K_{2}$ соответствует необходимой мощности электронного преобразователя:

$$
K_{2}=3 \frac{I_{\text {ampl,rated }}}{\sqrt{2}} \frac{U_{D C, \text { rated }}}{\sqrt{6}},
$$

где $I_{\text {ampl,rated }}$ и $U_{D C, \text { rated }}$ - требуемые ток и напряжение $\mathrm{AC} / \mathrm{DC}$ преобразователя при максимальной мощности генератора $(T=100 \%, n=100 \%)$.

Величину мощности AC/DC преобразователя (9) необходимо добавить в критерий оптимизации (8), поскольку в противном случае полученный коэффициент мощности генератора может быть низким, что негативно отразиться на стоимости DC/AC преобразователя.

В данной статье предполагается, что генератор работает на управляемый выпрямитель (AC/DC преобразователь) с IGBT-транзисторами, с широтноимпульсной модуляцией тока, система управления которого использует обратные связи по фазным то- кам и позиции ротора генератора $[16,17]$. В этом случае генератор формирует несинусоидальное напряжение, однако ток генератора поддерживается близким к синусоидальному.

Коэффициент $\quad K_{3}=L \cdot\left(h_{m a g}+0,001 \cdot m\right) \cdot\left(R_{1}-R_{2}\right) \quad$ представляет объем постоянных магнитов и используется для снижения стоимости магнитов; константа 0,001 м учитывает, что цена за единицу объема для тонких магнитов больше, чем для более толстых [26].

\section{Исследование исходной конструкции генератора}

На рис. 5 показано распределение магнитной индукции ГПП (конструкция до оптимизации). Благодаря эффекту концентрации потока магнитная индукция достигает значения 2 Тесла не только в ребрах, но и в некоторых зубцах статора. Таким образом, задача расчёта ГПП является крайне нелинейной, и возможность аппроксимации зависимости потерь от механической мощности полиномами низких степеней является неочевидной.

В табл. 7 приводятся величины потерь для отдельных точек 2-, 3- и 9-точечого профилей, а также средние величины потерь ГПП. Механические потери были приняты равными (3 ВТ) $\left(n / n_{\text {rated }}+\left[n / n_{\text {rated }}\right]^{3}\right)$. Средние потери по двухточечному и девятиточечному профилю практически совпадают (различие менее 0,03 Вт). Средние потери по трехточечному профилю отличаются от последних на 0,1 Вт. Чтобы убедиться, что совпадение численных значений средних потерь не случайно, на рис. 6, А символом «»» показаны точки 9-точечного профиля, а также парабола (квадратный трехчлен), аппроксимирующая эти точки. Эта же парабола показана на рис. 6 , Б. На рис. 6 , Б символом «•» показаны точки двухточечного профиля. Символом «+» показаны точки трехточечного профиля. Из рис. 6 видно, что все точки хорошо аппроксимируются данной параболой. Поэтому в этой работе оптимизация выполнена с использованием двухточечного замещающего профиля.

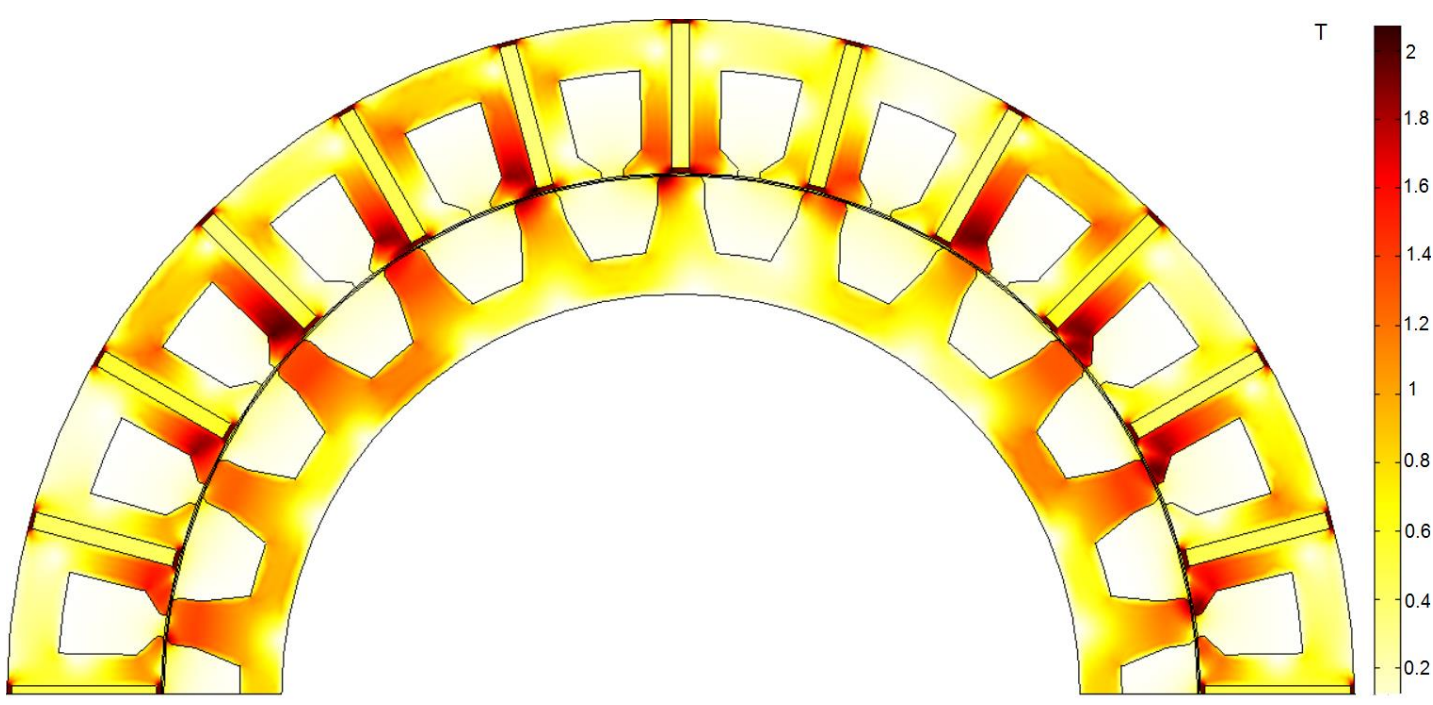

Рис. 5. Распределение магнитной индукиии исходной конструкиии генератора с переключением потока

Fig. 5. Flux density plot of the initial flux switching generator design 


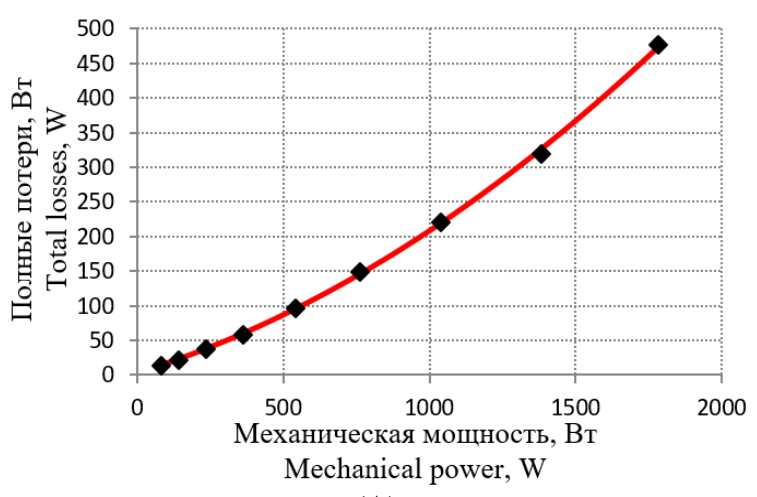

(A)

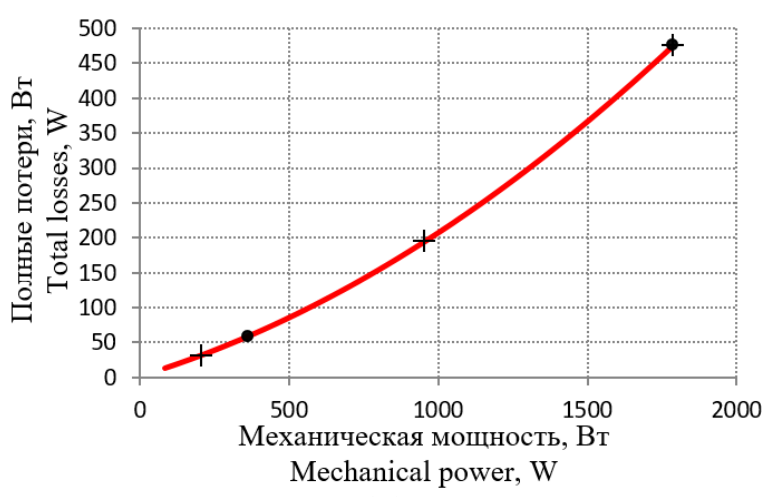

(Б)

Рис. 6. Рассчитанные потери исходной конструкиии генератора с переключением потока и их аппроксимачия полиномом второго порядка

Fig. 6. Calculated losses of the initial flux switching generator design and their approximation with quadric polynomial

Таблица 7. Рассчитанные потери исходной конструкичи генератора с переключением потока

Table 7. Calculated losses of the initial design of the flux switching generator

\begin{tabular}{|c|c|c|c|}
\hline \multicolumn{4}{|c|}{ Потери, Вт/Power losses, W } \\
\hline $\begin{array}{c}\text { Точка рабочего } \\
\text { Оperating mode, } i\end{array}$ & $\begin{array}{c}\text { 2-т. профиль } \\
\text { 2-mode } \\
\text { profile }\end{array}$ & $\begin{array}{c}\text { 3-т. профиль } \\
\text { 3-mode profile }\end{array}$ & $\begin{array}{c}\text { 9-т. профиль } \\
\text { 9-mode } \\
\text { profile }\end{array}$ \\
\hline 1 & 58,9 & 31,4 & 13,3 \\
\hline 2 & 476,6 & 196,5 & 21,7 \\
\hline 3 & - & 476,6 & 37,8 \\
\hline 4 & - & - & 57,8 \\
\hline 5 & - & - & 96,1 \\
\hline 6 & - & - & 148,1 \\
\hline 7 & - & - & 220,1 \\
\hline 8 & - & - & 319,3 \\
\hline 9 & 112 & 112,1 & 476,6 \\
\hline$\left\langle P_{\text {loss }}>\right.$ & & & 112 \\
\hline
\end{tabular}

\section{Результаты оптимизации}

В данной работе выполнена оптимизация, в соответствии с критерием (8), по двухточечному замещающему профилю. На рис. 7 показана геометрия опти- мизированной конструкции ГПП и магнитная индукция при максимальной мощности генератора. По сравнению с рис. 5 магнитная индукция величиной 2 Т не достигается, за исключением рёбер статора. Уменьшилась толщина ярем статора $R_{1}-R_{2}$. Ширина зубцов у основания сузилась. Ширина зубцов статора увеличивается ближе к зазору.

Рис. 8 поясняет закономерность этих результатов. Когда зубец ротора находится над половиной зубца статора, магнитный поток, втекающий в эту половину и представленный линиями магнитного поля $1,2,3,4$, замыкается через ярма и раздваивается на потоки 1, 2 и 3, 4. Для проведения таких потоков достаточна толщина ярма, примерно равная половине от половины зубца статора. Кроме того, поток 1, 2 равномерно вдоль магнита переходит на вторую часть магнитопровода зубца и практически полностью ослабляется у основания зубца. Поэтому толщина стали зубца, расположенная по одну сторону магнита, примерно равна толщине ярма. Поток в зубце, обусловленный пазовым рассеянием, меньше у основания паза, что также способствует трапециевидной форме зубца.

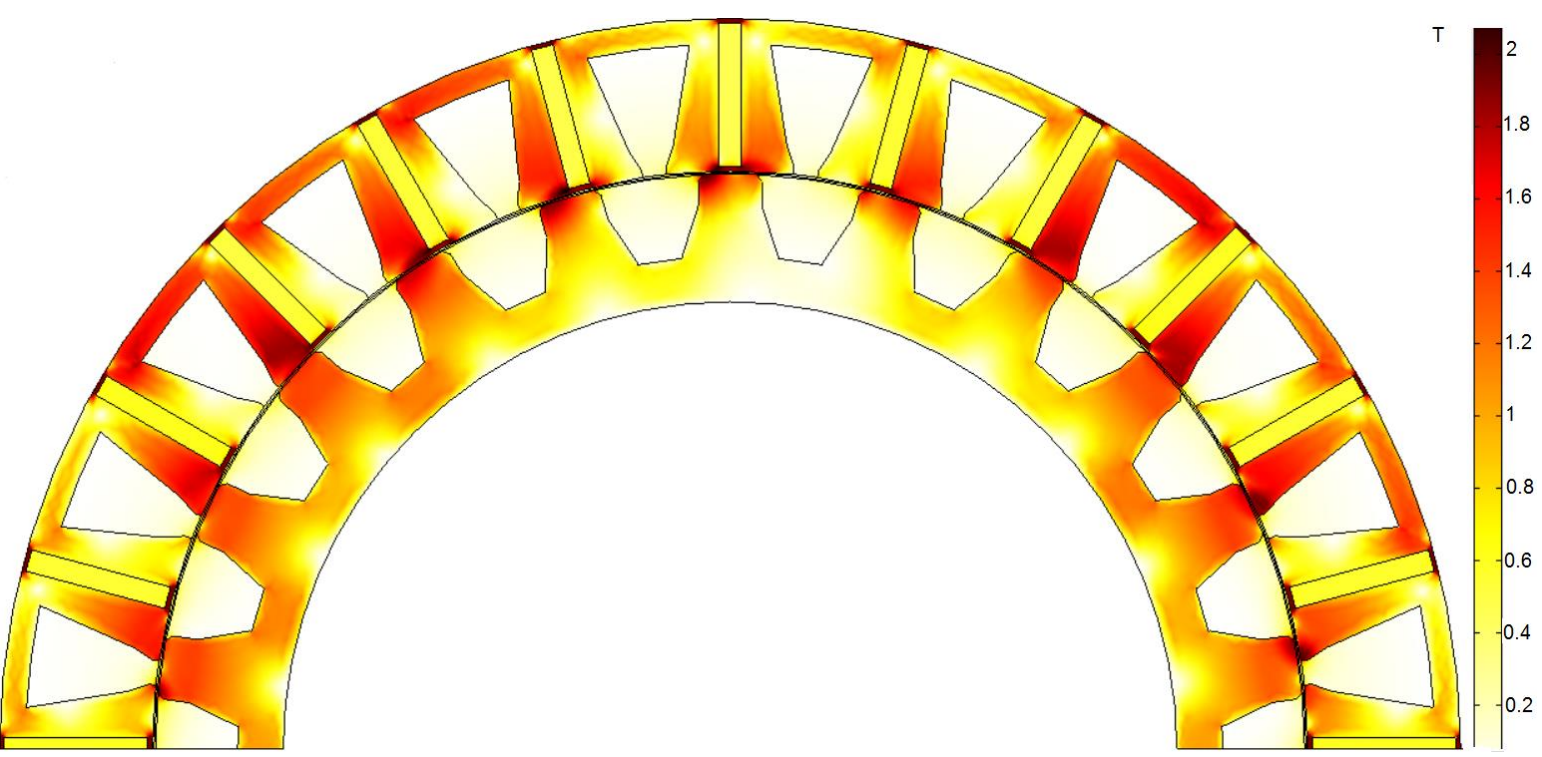

Pис. 7. Распределение магнитной индукиии оптимизированной конструкиии генератора с переключением потока

Fig. 7. Flux density plot of the optimized flux switching generator design 


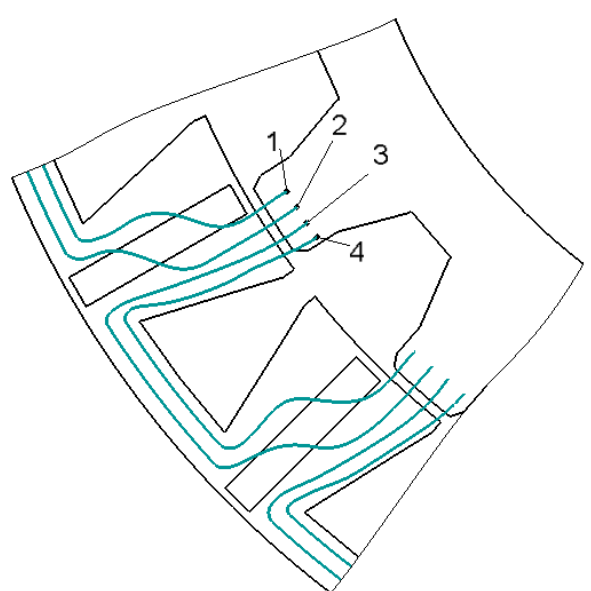

Pис. 8. Пути протекания магнитного потока в генераторе с переключением потока

Fig. 8. Flux flow in flux switching generator

Как упомянуто выше, зависимость $P_{\text {loss }}\left(P_{\text {mech }}\right)$ в начальном приближении хорошо описывается квадратным трехчленом. Чтобы убедиться в том, что потери в оптимальной конструкции ГПП также хорошо описываются квадратным трехчленом, для оптимальной конструкции был выполнен расчёт по 3- и 9точечным профилям.

Потери в различных точках цикла работы приведены в табл. 8. Хотя различия в результатах расчёта средних потерь для оптимизированной конструкции больше, чем для начального приближения, результаты расчётов все же оказываются очень близкими к друг другу. Рис. 9 аналогичен рис. 6 и демонстрирует, что квадратичная аппроксимация зависимости потерь от механической мощности для оптимизированной конструкции довольно точна, что подтверждает справедливость оптимизации по двухточечному профилю.

В табл. 9 изображены основные параметры ГПП до и после оптимизации.

Поскольку многокритериальная оптимизация должна обеспечить компромисс между величинами отдельных целевых параметров, уменьшение одного из них происходит за счет увеличения других. В результате оптимизации потери уменьшились на 30 \%, что соответствует увеличению КПД на 6 \%. Требуемая мощность преобразователя уменьшилась на $10 \%$. Однако это потребовало увеличения значения целевого параметра $K_{3}$ на 4 \%, что соответствует увеличению массы магнитов на 10 \%. При этом общая масса активных материалов машины снизилась. Также снизились пульсации момента и момент страгивания.

Таблица 8. Рассчитанные потери оптимизированной конструкции генератора с переключением потока

Table 8. Calculated losses of the optimized design of the flux switching generator

\begin{tabular}{|c|c|c|c|}
\hline \multicolumn{4}{|c|}{ Потери, Вт/Power losses, W } \\
\hline $\begin{array}{c}\text { Точка рабочего } \\
\text { цикла, } i\end{array}$ & $\begin{array}{c}\text { 2-т. профиль } \\
\text { 2-mode } \\
\text { profile }\end{array}$ & $\begin{array}{c}\text { 3-т. профиль } \\
\text { 3-mode } \\
\text { profile }\end{array}$ & $\begin{array}{c}\text { 9-т. профиль } \\
\text { 9-mode } \\
\text { profile }\end{array}$ \\
\hline 1 & 46,5 & 27,6 & 13,6 \\
\hline 2 & 302,2 & 135,1 & 20,4 \\
\hline 3 & - & 302,2 & 31,7 \\
\hline 4 & - & - & 46 \\
\hline 5 & - & - & 71,1 \\
\hline 6 & - & - & 104,5 \\
\hline 7 & - & - & 149,9 \\
\hline 8 & - & - & 210,8 \\
\hline 9 & 79 & 79,2 & 302,2 \\
\hline$<P_{\text {loss }}>$ & & & 78,8 \\
\hline
\end{tabular}

Таблица 9. Основные параметры ГПП до и после оптимизации

Table 9. The main parameters of the flux switching generator before and after optimization

\begin{tabular}{|c|c|c|}
\hline \multirow[b]{2}{*}{$\begin{array}{l}\text { Параметр } \\
\text { Parameter }\end{array}$} & \multicolumn{2}{|c|}{ Величина/Value } \\
\hline & $\begin{array}{l}\text { До оптимизации } \\
\text { Before optimization }\end{array}$ & $\begin{array}{l}\text { После оптимизации } \\
\text { After optimization }\end{array}$ \\
\hline Радиус дна паза статора $R_{2}, \mathrm{~mm} /$ Radius of the stator slot bottom $\mathrm{R}_{2}, \mathrm{~mm}$ & 74 & 77,2 \\
\hline Внутренний радиус статора $R_{3}$, мм/Inner radius of the stator $\mathrm{R}_{3}, \mathrm{~mm}$ & 61,7 & 63,3 \\
\hline Угловой размер паза статора $\alpha_{1}, t_{s} /$ Angular size of the stator slot $\alpha_{1}, t_{s}$ & 0,45 & 0,28 \\
\hline Угловой размер паза статора $\alpha_{2}, t_{s} /$ Angular size of the rotor slot $\alpha_{2}, t_{s}$ & 0,5 & 0,558 \\
\hline Толщина магнитов, мм/Width of the permanent magnets, mm & 2 & 2,41 \\
\hline $\begin{array}{l}\text { Угловой размер поверхности зубца ротора, обращенной к зазору, } t_{r} \\
\text { Angular size of the rotor tooth surface facing the air gap, } t_{r}\end{array}$ & 0,265 & 0,324 \\
\hline $\begin{array}{l}\text { Угол тока при максимальной нагрузке генератора, электрических радиан } \\
\text { Current angle at the maximum generator power, electrical radians }\end{array}$ & 0,1 & 0,086 \\
\hline Средние потери $K_{l}, \mathrm{BT} /$ Average losses $K_{1}, \mathrm{~W}$ & 112 & 78,8 \\
\hline $\begin{array}{l}\text { КПД про девятиточечному профилю, \% } \\
\text { Average efficiency when applying the nine-mode profile, } \%\end{array}$ & 79,4 & 85,5 \\
\hline $\begin{array}{l}\text { Требуемая номинальная мощность AC/DC преобразователя } K_{2}, \mathrm{BT} \\
\text { Required rated power of the AC/DC converter }\left(K_{2}\right), \mathrm{W}\end{array}$ & 1973 & 1767 \\
\hline$K_{3}=L \cdot\left(h_{m a g}+0,001 \cdot \mathrm{M}\right) \cdot\left(R_{1}-R_{3}\right), \mathrm{m}^{3}$ & $5,478 \times 10^{-6}$ & $5,71 \times 10^{-6}$ \\
\hline $\begin{array}{l}\text { Полные пульсации момента при максимальной мощности генератора, \% } \\
\text { Torque ripple at the maximum generator power, } \%\end{array}$ & 1,6 & 1,3 \\
\hline Момент страгивания, $\mathrm{H} \cdot \mathrm{M} /$ Cogging torque, $\mathrm{N} \cdot \mathrm{m}$ & 0,6 & 0,43 \\
\hline Масса магнитов, кг/Weight of the permanent magnets, $\mathrm{kg}$ & 0,613 & 0,674 \\
\hline Масса меди, кг/Weight of the copper, $\mathrm{kg}$ & 1 & 1,06 \\
\hline Масса стали статора, кг/Weight of the stator magnetic core, $\mathrm{kg}$ & 3,88 & 3,26 \\
\hline Масса стали ротора, кг/Weight of the rotor magnetic core, $\mathrm{kg}$ & 2,17 & 2,46 \\
\hline Целевая функция $F, \mathrm{BT}^{3} \cdot \mathrm{M}^{3 / 2} /$ Objective function $F, \mathrm{~W}^{3} \cdot \mathrm{m}^{3 / 2}$ & 1881 & 1087 \\
\hline Масса активных материалов, кг/Weight of the active materials, $\mathrm{kg}$ & 7,66 & 7,45 \\
\hline
\end{tabular}




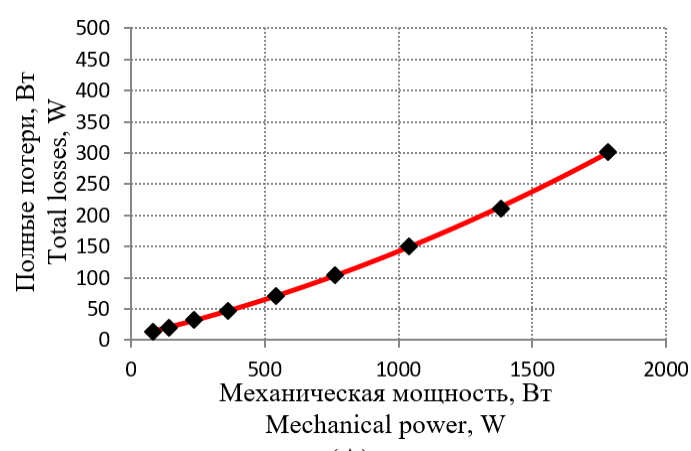

(A)

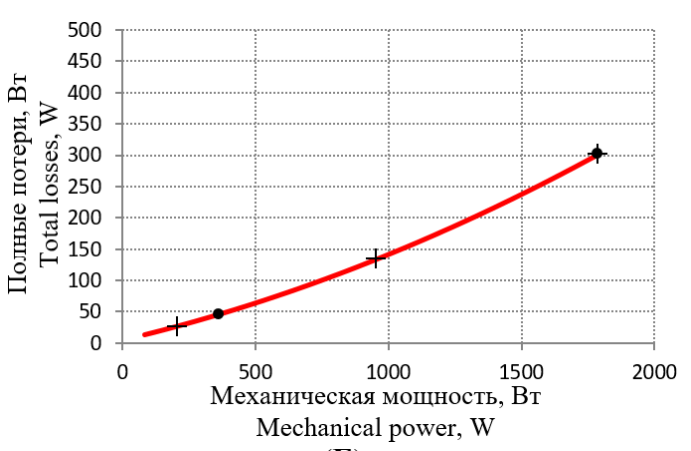

(Б)

Pис. 9. Рассчитанные потери оптимизированной конструкции генератора с переключением потока и их аппроксимация полиномом второго порядка

Fig. 9. Calculated losses of the optimized flux switching generator design and their approximation with quadric polynomial

\section{Заключение}

Описана процедура оптимизации безредукторного генератора с переключением потока (ГПП) для ветряной турбины. Средние потери, требуемая мощность преобразователя и параметр $K_{3}$, учитывающий стоимость постоянных магнитов, были выбраны в качестве критериев оптимизации. Целевой параметр $K_{3}$ выбран так, чтобы учесть, что тонкие магниты являются более дорогостоящими, чем более толстые.

Для снижения вычисленных затрат при оптимизации предложен метод расчета замещающих профилей. 2- и 3-точечные профили были составлены на основе исходного 9-ти точечного профиля. Потери ГПП, вычисленные с помощью 2-, 3- и 9-точечных профилей, совпадают с большой точностью, что позволяет использовать для оптимизационных расчетов профиль с меньшим количеством точек.

\section{СПИСОК ЛИТЕРАТУРЫ}

1. Григораш О.В., Кривошей А.А., Смык В.В. Автономные гибридные электростанции // Политематический сетевой электронный научный журнал Кубанского государственного аграрного университета. - 2014. - № 10. - С. 1-12.

2. Li H., Chen Z. Overview of different wind generator systems and their comparisons // IET Renewable Power Generation. - 2007. V. 2 - № 5. - P. 123-138. DOI: 10.1049/iet-rpg:20070044.

3. Energy cost estimation of small wind power systems - an integrated approach / S. Xu, R. Shao, L. Chang, C. Church // IEEE Journal of Emerging and Selected Topics in Power Electronics. - 2015. - V. 3. № 4. - P. 945-956. DOI: 10.1109/JESTPE.2015.2444398.

4. Comparison of direct-drive and geared generator concepts for wind turbines / H. Polinder, F. Pijl, G. Vilder, P. Tavner // IEEE Transactions on Energy Conversion. - 2006. - V. 21. - № 3. P. 725-733. DOI: 10.1109/TEC.2006.875476.

5. Optimal design of direct-driven PM wind generator for maximum annual energy production / S. Jung, H. Jung, S. Hahn, H.K. Jung, C. Lee // IEEE Transactions on Magnetics. - 2008. - V. 44. - № 6. P. 1318-1338. DOI: 10.1109/TMAG.2007.916250.

6. Optimal design of permanent magnet synchronous generator for wind energy conversion considering annual energy input and magnet volume / J. Faiz, B. Ebrahimi, M. Rajabi-Sebdani, A. Khan // Proc. of 2009 International Conference on Sustainable Power Generation and Supply. - Nanjing, China, April 6-7, 2009. - P. 1-6. DOI: 10.1109/SUPERGEN.2009.5348100.

7. Isfahani A., Boroujerdi A., Hasanzadeh S. Multi-objective design optimization of a large-scale direct-drive permanent magnet generator for wind energy conversion systems // Frontiers in Energy. 2014. - V. 8. - № 2. - P. 182-191. DOI: 10.1007/s11708014-0320-z.
Поскольку многокритериальная оптимизация должна обеспечить компромисс между величинами отдельных целевых параметров, уменьшение одного из них может произойти за счет увеличения других. В результате оптимизации потери уменьшились на $30 \%$, что соответствует увеличению КПД на 6 \%. Требуемая мощность преобразователя уменьшилась на $10 \%$. Однако это потребовало увеличения значения целевого параметра $K_{3}$ на $4 \%$, что соответствует увеличению массы магнитов на 10 \%. При этом общая масса активных материалов машины снизилась. Также снизились зубцовые и полные пульсации момента.

В процессе оптимизации были найдены некоторые предпочтительные геометрические особенности конструкции ГПП, среди них - трапецеидальная форма зубца статоpa, толщина которого уменьшается ближе к основанию.

Результаты работы получены при поддержке средствами МОН РФ, задание № 8.9549.2017/8.9.

8. Vahedi A., Meo S., Zohoori A. An AHP-based approach for design optimization of flux-switching permanent magnet generator for wind turbine applications // International Transactions on Electrical Energy Systems. 2016. - V. 26. - № 1. - P. 1318-1338. DOI: $10.1002 /$ etep.2149.

9. A new outer-rotor flux switching permanent magnet generator for wind farm applications / A. Zohoori, A. Vahedi, M. Noroozi, S. Meo // Wind Energy. - 2017. - V. 20. - № 1. - P. 3-17. DOI: 10.1002/we.1986.

10. Soleimani J., Ejlali A., Moradkhani M. Flux switching permanent magnet generator design and optimization using response surface methodology // Periodicals of Engineering and Natural Sciences. - 2019. - V. 7. - № 1. P. 54-66. DOI: dx.doi.org/10.21533/pen.v7i1.231.g215.

11. Design of a flux-switching electrical generator for wind turbine systems / J. Ojeda, M. Simões, G. Li, M. Gabsi // IEEE Transactions on Industry Applications. - 2012. - V. 48. - № 6. P. 1808-1816. DOI: 10.1109/TIA.2012.2221674.

12. Конечноэлементная модель электрической машины с переключением потока для исследования динамических режимов работы / В.А. Дмитриевский, В.А. Прахт, Ф.Н. Сарапулов, В.А. Климарев // Электротехника. - 2012. - № 3. - С. 7-13.

13. Low cost flux-switching brushless AC machines / J. Chen, Z. Zhu, S. Iwasaki, R. Deodhar // Proc. of 2010 IEEE Vehicle Power and Propulsion Conference. - Lille, France, September 1-3, 2010. P. 1-6. DOI: 10.1109/VPPC.2010.5728984.

14. Anandavel P., Rajambal K., Chellamuthu C. Power optimization in a grid-connected wind energy conversion system // Proc. of 2005 International Conference on Power Electronics and Drives Systems. - Kuala Lumpur, Malaysia, November 28 - December 1, 2005. - P. 1617-1621. DOI: 10.1109/PEDS.2005.1619947.

15. Pishgar-Komleh S., Keyhani A., Sefeedpari P. Wind speed and power density analysis based on Weibull and Rayleigh 
distributions (a case study: Firouzkooh county of Iran) // Renewable and Sustainable Energy Reviews. 2015. - V. 42. № 1. - P. 313-322. DOI: doi.org/10.1016/j.rser.2014.10.028a.

16. Akuru U., Kamper M. Intriguing behavioral characteristics of rareearth-free flux switching wind generators at small- and large-scale power levels // IEEE Transactions on Industry Applications. 2018. V. 54. - № 6. - P. 5772-5782. DOI: 10.1109/TIA.2018.2848979

17. Analysis of electromagnetic performance of flux-switching permanentmagnet machines by nonlinear adaptive lumped parameter magnetic circuit model / Z. Zhu, Y. Pang, D. Howe, S. Iwasaki, R. Deodhar, A. Pride // IEEE Transactions on Magnetics. 2005. - V. 41. - № 11. P. 4277-4287. DOI: 10.1109/TMAG.2005.854441

18. Wang Y., Deng Z. A position sensorless method for direct torque control with space vector modulation of hybrid excitation fluxswitching generator // IEEE Transactions on Energy Conversion 2012. - V. 27. - № 4. - P. 912-921. DOI: 10.1109/TEC.2012.2210718

19. Dmitriveskii V., Prakht V., Kazakbaev V. IE5 energy-efficiency class synchronous reluctance motor with fractional slot winding // IEEE Transactions on Industry Applications. 2019. - V. 55. № 5. - P. 4676-4684. DOI: 10.1109/TIA.2019.2924405.

20. Dmitrievskii V., Prakht V., Kazakbaev V. Optimal design of a highspeed single-phase flux reversal motor for vacuum cleaners // Energies. 2018. - V. 11. - № 12. - P. 1-13. DOI: 10.3390/en11123334.

21. Ruoho S., Dlala E., Arkkio A. Comparison of demagnetization models for finite-element analysis of permanent-magnet synchronous machines // IEEE Transactions on Magnetics. - 2007. V. 43. - № 11. - P. 3964-3968. DOI: 10.1109/TMAG.2007.906749

22. Efficiency calculation of wind turbine generation system with doubly-fed induction generator / R. Takahashi, H. Ichita, J. Tamura, M. Kimura, M. Ichinose, M. Futami, K. Ide // Proc. of XIX International Conference on Electrical Machines (ICEM 2010). - Rome, Italy, September 6-8, 2010. - P. 1-4. DOI: 10.1109/ICELMACH.2010.56083.

23. Виноградов А.Б. Векторное управление электроприводами переменного тока. - Иваново: Ивановский государственный энергетический университет имени В.И. Ленина, 2008. - 298 с.

24. Cupertino F., Pellegrino G., Gerada C. Design of synchronous reluctance machines with multiobjective optimization algorithms // IEEE Transactions on Industry Applications. - 2014. - V. 50. № 6. - P. 3617-3627. DOI: 10.1109/ECCE.2013.6646934.

25. Kamper M., Merwe F., Williamson S. Direct finite element design optimization of the cageless reluctance synchronous machine // IEEE Transactions on Energy Conversion. - 1996. - V. 11. № 3. - P. 547-555. DOI: $10.1109 / 60.537006$.

26. Chen Yang. NdFeB magnets, price list of standard block magnets. URL: http://www.ndfebmagnets.de/CY-PriceList-NdFeB-Block.pdf (дата обращения 09.01.2020).

Поступила 31.08.2019.

\section{Информация об авторах}

Прахт В.A., кандидат технических наук, доцент кафедры электротехники и электротехнологических систем Уральского Энергетического Института Уральского Федерального Университета.

Дмитриевский В.А., кандидат технических наук, доцент кафедры электротехники и электротехнологических систем Уральского Энергетического Института Уральского Федерального Университета.

Казакбаев В.M., кандидат технических наук, младший научный сотрудник кафедры электротехники и электротехнологических систем Уральского Энергетического Института Уральского Федерального Университета. 
UDC 621.313.322

\title{
OPTIMIZATION OF GEARLESS FLUX SWITCHING MACHINE DESIGN FOR WIND GENERATOR SYSTEMS
}

\author{
Vladimir A. Prakht 1 , \\ va.prakht@urfu.ru
}

\author{
Vladimir A. Dmitrievskii ${ }^{1}$ \\ vladimir.dmitrievsky@urfu.ru \\ Vadim M. Kazakbaev1, \\ vadim.kazakbaev@urfu.ru \\ 1 Ural Federal University,
19, Mira street, Ekaterinburg, 620002, Russia.
}

The relevance of the research is caused by the increasing need for autonomous hybrid power plants using renewable sources to reduce the consumption of fossil fuels. Improving the performance and reducing the cost of gearless wind generators used in such power plants will contribute to improvement of technical and economic characteristics of the plants.

The main aim of the research includes the elaboration of an effective approach for optimizing the flux switching generator for gearless wind turbines, which reduces the use of computing resources, in comparison with existing approaches; elaboration of recommendations for the design of the flux switching wind generator with permanent magnets on the stator; improving the initial design of the generator.

Objects of the research are the mathematical model and the design of flux switching generator with permanent magnets on the stator. Methods: assessment and analysis of wind speed data worldwide; derivative-free optimization method; finite element method; mathematical modeling; statistical methods.

Results. The multicriteria optimization of the design of a gearless wind flux switching generator was carried out. As a result, the generator efficiency was increased, its torque ripple and the rated power of the electronic converter of the wind turbine system were reduced. General recommendations regarding the geometry are obtained which can be used for designing similar flux switching generators. $A$ method is proposed for construction of substituting profiles of the operating characteristics of wind turbines, which can be used to reduce computational effort during the optimization. The computational cost reduction using the obtained method is demonstrated using the example of replacing the original nine-point working profile of a wind turbine with a substituting two-point one.

\section{Key words:}

Autonomous hybrid power plants, direct driven generators, wind generators, wind power, renewable energy sources, permanent magnet generators, optimal design, switching flux machine, energy efficiency.

The research was conducted on theme no. 8.9549.2017/8.9 within the frame of the government task of the Ministry of Education and Science of the Russian Federation in $R \& D$.

\section{REFERENCES}

1. Grigorash O.V., Krivoshey A.A., Smyk V.V. Autonomous hybrid power plants. Avtonomnyye gibridnyye elektrostantsii. Scientific Journal of Kuban state agrarian University, 2014, no. 10, pp. 1-12. In Rus.

2. Li H., Chen Z. Overview of different wind generator systems and their comparisons. IET Renewable Power Generation, 2007, vol. 2 no. 2, pp. 123-138. DOI: 10.1049/iet-rpg:20070044.

3. Xu S., Shao R., Chang L., Church C. Energy cost estimation of small wind power systems - an integrated approach. EEE Journal of Emerging and Selected Topics in Power Electronics, 2015, vol. 3, no. 4. DOI: 10.1109/JESTPE.2015.2444398

4. Polinder H., Pijl F., Vilder G., Tavner P. Comparison of directdrive and geared generator concepts for wind turbines. IEEE Transactions on Energy Conversion, 2006, vol. 21, no. 3, pp. 725-733. DOI: 10.1109/TEC.2006.875476.

5. Jung S., Jung H., Hahn S., Jung H., Lee C. Optimal design of direct-driven PM wind generator for maximum annual energy production. IEEE Transactions on Magnetics, 2008, vol. 44, no. 6, pp. 1318-1338. DOI: 10.1109/TMAG.2007.916250.

6. Faiz J., Ebrahimi B., Rajabi-Sebdani M., Khan A. Optimal design of permanent magnet synchronous generator for wind energy conversion considering annual energy input and magnet volume. Proc of 2009 International Conference on Sustainable Power Generation and Supply. Nanjing, China, 6-7 April 2009. pp. 1-6. DOI: 10.1109/SUPERGEN.2009.5348100.

7. Isfahani A., Boroujerdi A., Hasanzadeh S. Multi-objective design optimization of a large-scale direct-drive permanent magnet gen- erator for wind energy conversion systems. Frontiers in Energy, 2014, vol. 8, no. 2, pp. 182-191. DOI: 10.1007/s11708-014-0320-z.

8. Vahedi A., Meo S., Zohoori A. An AHP-based approach for design optimization of flux-switching permanent magnet generator for wind turbine applications. International Transactions on Electrical Energy Systems, 2016, vol. 26, no. 1, pp. 1318-1338. DOI: 10.1002/etep.2149.

9. Zohoori A., Vahedi A., Noroozi M., Meo S. A new outer-rotor flux switching permanent magnet generator for wind farm applications. Wind Energy, 2017, vol. 20, no. 1, pp. 3-17. DOI: $10.1002 /$ we. 1986.

10. Soleimani J., Ejlali A., Moradkhani M. Flux switching permanent magnet generator design and optimization using response surface methodology. Periodicals of Engineering and Natural Sciences, 2019, vol. 7, no. 1, pp. 54-66. DOI: dx.doi.org/10.21533/pen.v7i1.231.g215.

11. Ojeda J., Simões M., Li G., Gabsi M. Design of a flux-switching electrical generator for wind turbine systems. IEEE Transactions on Industry Applications, 2012, vol. 48, no. 6, pp. 1808-1816. DOI: $10.1109 /$ TIA.2012.2221674

12. Dmitrievskii V., Prakht, V., Sarapulov F., Klimarev V. A finite element model of electric machine with flux switching-over for studying the dynamic operation modes. Russian Electrical Engineering, 2012, vol. 83, no. 2, pp. 126-131. DOI: doi.org/10.3103/S1068371212030042.

13. Chen J., Zhu Z., Iwasaki S., Deodhar R. Low cost flux-switching brushless AC machines. Proc. of 2010 IEEE Vehicle Power and Propulsion Conference. Lille, France, 1-3 Sept. 2010. pp. 1-6. DOI: $10.1109 /$ VPPC. 2010.5728984 
14. Anandavel P., Rajambal K., Chellamuthu C. Power optimization in a grid-connected wind energy conversion system. Proc. of 2005 International Conference on Power Electronics and Drives Systems. Kuala Lumpur, Malaysia, 28 Nov. - 1 Dec. 2005 DOI: 10.1109/PEDS.2005.1619947.

15. Pishgar-Komleh S., Keyhani A., Sefeedpari P. Wind speed and power density analysis based on Weibull and Rayleigh distributions (a case study: Firouzkooh county of Iran). Renewable and Sustainable Energy Reviews, 2015, vol. 42, no. 1, pp. 313-322. DOI: doi.org/10.1016/j.rser.2014.10.028a.

16. Akuru U., Kamper M. Intriguing behavioral characteristics of rareearth-free flux switching wind generators at small- and large-scale power levels. IEEE Transactions on Industry Applications, 2018, vol. 54, no. 6, pp. 5772-5782. DOI: 10.1109/TIA.2018.2848979.

17. Zhu Z., Pang Y., Howe D., Iwasaki S., Deodhar R., Pride A. Analysis of electromagnetic performance of flux-switching permanentmagnet machines by nonlinear adaptive lumped parameter magnetic circuit model. IEEE Transactions on Magnetics, 2005, vol. 41, no. 11, pp. 4277-4287. DOI: doi.org: 10.1109/TMAG.2005.854441.

18. Wang Y., Deng Z. A position sensorless method for direct torque control with space vector modulation of hybrid excitation fluxswitching generator. IEEE Transactions on Energy Conversion, 2012, vol. 27, no. 4, pp. 912-921. DOI: 10.1109/TEC.2012.2210718

19. Dmitriveskii V., Prakht V., Kazakbaev V. IE5 energy-efficiency class synchronous reluctance motor with fractional slot winding. IEEE Transactions on Industry Applications, 2019, vol. 55, no. 5, pp. 4676-4684. DOI: 10.1109/TIA.2019.2924405.

20. Dmitrievskii V., Prakht V., Kazakbaev V. Optimal design of a highspeed single-phase flux reversal motor for vacuum cleaners. Energies, 2018, vol. 11, no. 12, pp. 1-13, DOI: 10.3390/en11123334.

\section{Information about the authors}

Vladimir A. Prakht, Cand. Sc., associate professor, Ural Federal University.

Vladimir A. Dmitrievskii, Cand Sc., associate professor, Ural Federal University.

Vadim M. Kazakbaev, Cand Sc., junior researcher, Ural Federal University.
21. Ruoho S., Dlala E., Arkkio A. Comparison of demagnetization models for finite-element analysis of permanent-magnet synchronous machines. IEEE Transactions on Magnetics, 2007, vol. 43, no. 11, pp. 3964-3968. DOI: 10.1109/TMAG.2007.906749.

22. Takahashi R., Ichita H., Tamura J., Kimura M., Ichinose M., Futami M., Ide K. Efficiency calculation of wind turbine generation system with doubly-fed induction generator. Proc. of XIX International Conference on Electrical Machines (ICEM 2010). Rome, Italy, 6-8 Sept. 2010. DOI: 10.1109/ICELMACH.2010.56083.

23. Vinogradov A.B. Vektornoe upravlenie elektroprivodami peremennogo toka [Vector control of AC drives]. Ivanovo, Ivanovo State Power Engineering University named after V.I. Lenin (ISPU), 2008. $298 \mathrm{p}$

24. Cupertino F., Pellegrino G., Gerada C. Design of synchronous reluctance machines with multiobjective optimization algorithms. IEEE Transactions on Industry Applications, 2014, vol. 50, no. 6, pp. 3617-3627. DOI: 10.1109/ECCE.2013.6646934

25. Kamper M., Merwe F., Williamson S. Direct finite element design optimization of the cageless reluctance synchronous machine. IEEE Transactions on Energy Conversion, 1996, vol. 11, no. 3 , pp. 547-555. DOI: 10.1109/60.537006

26. Chen Yang. NdFeB magnets, price list of standard block magnets. Catalogue. Available at: http://www.ndfebmagnets.de/CYPriceList-NdFeB-Block.pdf (accessed 9 January 2020).

Received: 31.08.2019. 